\section{Effects of Electrosurgery on the Gingival Attachment in Rhesus Monkeys}

by

Norman R. Wilhelmsen, D.D.S., M.S.*

Sigurd P. RamfJord, L.D.S., PH.D. $\dagger$

JOHN R. BLANKENSHIP, D.D.S., M.S.

ELECTROSURGERY HAS BEEN recommended as an expedient and safe adjunct to impression techniques in restorative dentistry. ${ }^{1-3}$ The healing pattern of electrosurgical soft tissue incisions has been reported to resemble closely the healing following conventional surgery. ${ }^{4-7}$ However, the effect of electrosurgery on the attachment level of the gingival tissues in the absence or presence of periodontal disease is highly controversial. Inadequately substantiated claims vary from no loss, ${ }^{1,4-12}$ to extensive lowering of attachment levels postsurgically. ${ }^{13-15}$ It is generally assumed that electrosurgery used in restorative dentistry in the manner commonly recommended, is a safe procedure, ${ }^{1}$ but this assumption is only a hypothesis which never has been validated under controlled experimental conditions. Current knowledge is very sketchy with regard to the effects of contact of the activated electrosurgical needle with enamel or cementum. Of special concern is interference with formation of new epithelial and connective tissue attachments during the postsurgical healing.

Any questions regarding safety of a surgical procedure should be tested in animals both for ethical and practical reasons. Besides accurate histometrical measurements, animal studies also offer the opportunity for histologic evaluation. An investigation of the clinical and histological effects of gingival electrosurgery therefore was initiated in Rhesus monkeys which generally are accepted as choice animals for periodontal healing studies.

\section{Materials AND Methods}

Two female and two male Rhesus monkeys were used for the study. Three of the animals had complete adult dentitions. In the fourth animal the maxillary third molars were unerupted and the mandibular third molars were in the process of penetiating the mucosa. The molars that were present showed light to advanced

* Fort Lauderdale, Florida. Formerly, Department of Periodontics, The University of Michigan School of Dentistry, An n Arbc:, Michigan 48104.

† Department of Periodontics, The University of Michigan School of Dentistry, and Veterans Administration Hospital, Ann Arbor, Michigan 48104.

$\ddagger$ Veterans Administration Hospital, Ann Arbor, Michigan 48104. attrition. All of the animals had moderate supra and subgingival calculus with mild to moderate gingivitis. The three older animals showed slight apical migration of the epithelial attachment, mainly around the first molars and the anterior teeth; however, this usually did not exceed a distance of $1 \mathrm{~mm}$ from the cemento-enamel junction. In the youngest monkey with the unerupted third molars, the epithelial attachment was confined to the enamel. The monkeys weighed from 10 pounds for the youngest animal to 24 pounds for one of the older monkeys. They were maintained on a diet of Purina monkey chow§ and water ad libitum throughout the duration of the study.

The animals were allowed approximately 2 months of adaptation to the environment of their quarters before the experimental procedures were started. All teeth were scaled 4 to 10 weeks before the experiment. A few days prior to surgery the animals were anesthetized and full mouth impressions and color photographs were obtained. A horizontal groove was cut on the buccal aspect of the crowns of the teeth, approximately $1 \mathrm{~mm}$ coronally to the gingival margin. Measurements of the distances from the groove to the gingival margin and from the groove to the bottom of the gingival crevice were obtained using a blunt silver point, cotton forceps, and a Boley gauge.

In two animals the maxillary right and mandibular left quadrants were designated as experimental units; for the other two animals the maxillary left and mandibular right quadrants were designated experimental. The remaining quadrants were used as controls. An electrosurgical unit 9 which is supposed to deliver a fully rectified high frequency current of undamped wave form was employed to perform gingival electrosurgery around the teeth in the experimental quadrants. A straight single filament electrode tip** was bent to make a right angle with the surface of the gingiva and the depths of the crevices were measured with the inactivated electrode.

The first gingival incision was made close to the free gingival margin pointing the electrode in an acute angle with the tooth and with the tip of the instrument touching the tooth. The incision was aimed at the bottom of the gingival sulcus as predetermined by the probing. In order to make the tip contact the tooth and without bending the needle to the tooth contour; this incision tended to resect a thin wedge of gingival tissue including the gingival crest and the crevicular lining. A second incision was made with the side of the needle contacting the surface of the tooth in the crevicular area and extending the tip to the bottom of the crevice as previously located by the probe. The electric current was adjusted to the lowest setting that allowed for cutting without appreciable resistance to the electrode tip. The rheostat setting varies a little from one machine to another. In this instance a setting of 4

\footnotetext{
§ Ralston Purina Compaiı, , St. Louis, Missouri.

T Coles Radiosurg Electronic Scalpel IV, Clev Dent Division of Cavitron Corporation, Cleveland, Ohio.

** No. E-1-A Coles electrode for electrosurgery.
} 
had to be used. In clinical use of these machines the setting usually has to be between 3 and 4, depending to a certain extent on the voltage. The incisions were made with a steady and fairly fast movement and the electrode was not allowed to hesitate or dissipate heat in any one area. Each incision was made only once and never repeated. Great care was observed to avoid deep penetration of the electrode toward the alveolar crest. The electrosurgery was done by a person who has worked with this type of electrosurgical units for more than 25 years. ${ }^{16}$ The operated areas were permitted to heal without the use of dressings.

Immediately prior to sacrifice, clinical photographs and all measurements were repeated. The experimental time intervals were respectively 1 month, 3 months, $61 / 2$ months, and 9 months. The 9 month animal was the youngest and had the healthiest periodontal tissues. Following sacrifice the jaws were fixed in $10 \%$ neutral formalin for three weeks. The reference grooves in the teeth were cut deeper into the dentin, the jaws were divided into small sections, were decalcified in $20 \%$ formic acid, and embedded in paraffin. Buccal-lingual sections were cut at $7 \mu$ and stained with hematoxylin and eosin.

\section{Method of Sample Selection}

At the onset, it was decided to eliminate measurements from all cuspids and mandibular first bicuspids because of the anatomical differences of these teeth between humans and monkeys and the difficulty in attaining reproducible measurements. Third molars were eliminated because limited access would compromise the accuracy of the clinical portion of the study.

Clinical measurements were taken in duplicate on each animal at the midpoint of the buccal surfaces of the experimental and control teeth prior to surgery and prior to sacrifice. Histometric measurements were made on the buccal and lingual surfaces of the same teeth. Histometric measurements were recorded for each tooth from five central sections with some distance between them. No sections with artifactual distortion in the measurement areas were used.

\section{Measurement Technique}

Two biometric (clinical) measurements and five histometric measurements as outlined in Figure 1 were made for each tooth using the following code:

A. The distance from the apical surface of the horizontal groove to the free gingival margin at the mesiodistal midpoint of the facial surface of the tooth.

B. The distance from the apical surface of the horizontal groove to the bottom of the gingival crevice at the mesiodistal midpoint of the facial surface of the tooth. The initial clinical measurements were made prior to the surgery and the second set of measurements were made immediately prior to the

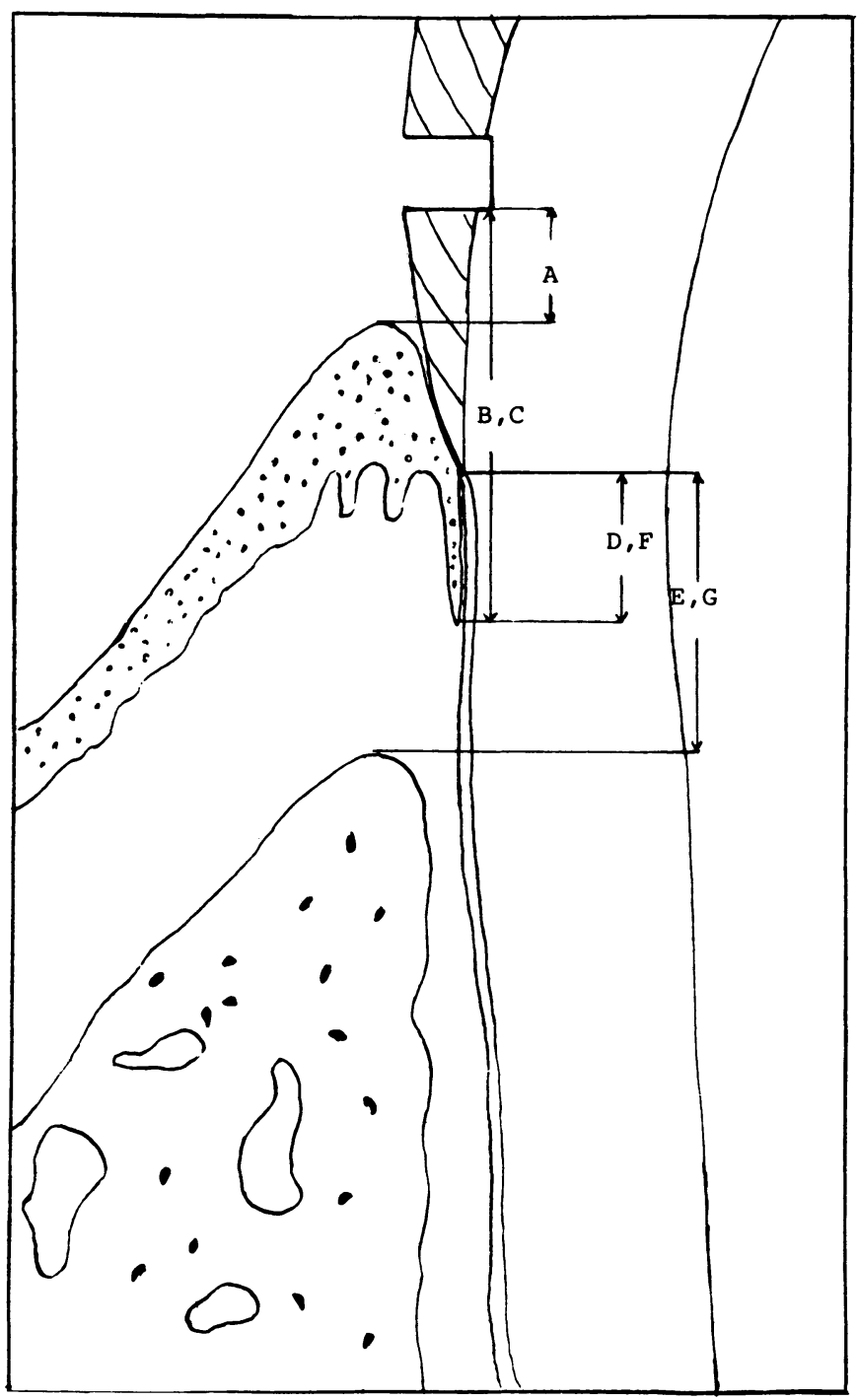

Figure 1. A diagram illustrating the linear measurements. For explanation see text.

sacrifice of the animals. Duplicate sets of measurements were taken to enhance measurement accuracy.

Histometric measurements were made from five tissue sections (not consecutive) for each location on each tooth. The following code (Fig. 1) was used:

C. The distance from the apical surface of the horizontal groove on the facial surface to the connective tissue border of the most apically located epithelial cells on the root surface.

D. The distance from the cemento-enamel junction on the facial surface to the connective tissue border of the most apically located epithelial cells on the root surface.

E. The distance from the cemento-enamel junction on the facial surface to the most coronal projection of the alveolar crest.

F. The distance from the cemento-enamel junction on the lingual surface to the connective tissue border of the most apically located epithelial cells on the root surface. 
G. The distance from the cemento-enamel junction on the lingual surface to the most coronal projection of the alveolar crest.

A silver point, shortened to provide an end diameter of $0.4 \mathrm{~mm}$ was held adjacent to the free gingival margin for one clinical measurement and was inserted to the bottom of the crevice for the other. In each location the silver point was grasped with a cotton forceps, the beaks of which were inserted into the horizontal groove in the tooth. Upon removal from the mouth the distance between the end of the silver point and the forceps was measured with a Boley gauge.

The silver point was inserted into the gingival crevice to the depth at which clinical resistance was felt.

For the histometric portion of the study a screw micrometer eyepiece* was used with which the longitudinal axis of measurement could be accurately paralleled with the dentin surface. The eyepiece furthermore allowed very precise measurements since the linear distance could be read directly from an adjustable micrometer drum.

\section{RESUlTS}

\section{Clinical Apprearance}

One Month Experimental Animal. The gingiva was red and appeared to be incompletely healed in the experimental areas (Fig. 2). Light probing provoked bleeding, especially around the experimental maxillary central and lateral incisors. The gingiva had a soft consistency and recession was apparent in the areas of the surgery.

Three Month Experimental Animal. The healed gingiva had a red hue at the maxillary bicuspids and the mandibular central and lateral incisors. At the experimental mandibular second bicuspid the gingiva was bright red. In other areas the healed gingiva had a fairly normal color, with evidence of a mild marginal gingivitis. The papilla between the nonoperated maxillary central and lateral incisors was very bulbous and red associated with accumulation of plaque and calculus.

Considerable gingival recession was observed in the operated areas. The recession was most noticeable at the maxillary and mandibular incisors and the mandibular bicuspids. The interdental papillae were blunted and bulbous. The buccal free gingiva was rounded and thick.

The healed gingiva was soft and edematous associated with the bulbous papillae whereas in other areas the gingiva had a more normal appearance and texture.

A sequestrum encircled the mandibular second bicuspid and extended coronally to the free gingival margin. This tooth was more mobile than normal. The buccal furcations were partially exposed on the operated mandibular bicuspids.

Six and One-Half Month Experimental Animal. The

\footnotetext{
*Oknor screw micrometer eyepiece Mi.T. 47, Ernest Leitz GMBH Wetzlar, Germany.
}

gingival color approached normal in most operated areas, although there was marginal gingivitis associated with accumulation of calculus on the labial surfaces of the operated mandibular anterior teeth.

Considerable gingival recession appeared in the anterior areas where electrosurgery had been performed (Fig. $3)$. This was most noticeable on the labial and palatal aspects of the maxillary incisors. The gingiva on the anterior labial surfaces had a more rolled appearance in the operated than in the control areas.

The gingiva in the experimental areas was nearly normal in firmness, but a slight marginal gingivitis was observed associated with plaque and calculus.

Nine Month Experimental Animal. The gingival color was nearly normal in the posterior operated areas. A blue hue was seen in the gingiva associated with the mandibular incisors, where there also was an increased bleeding potential. There was more calculus in the experimental quadrants than in the controls.

Considerable gingival recession was apparent in the anterior operated areas, particularly in the mandible where the gingiva had a rolled edematous appearance associated with abundant calculus and plaque on the labial surfaces of the operated mandibular anterior teeth.

The gingival contour at the posterior experimental teeth was nearly normal, but the gingival margin was at a more apical level than for the control teeth. The density was fairly normal except for softness around the mandibular anterior experimental teeth.

\section{Histological Observations}

The characteristic histologic findings are reported separately for each of the experimental animals.

One Month Experimental Animal. The connective tissue attachment level was at the cemento-enamel junction for some of the experimental as well as most of

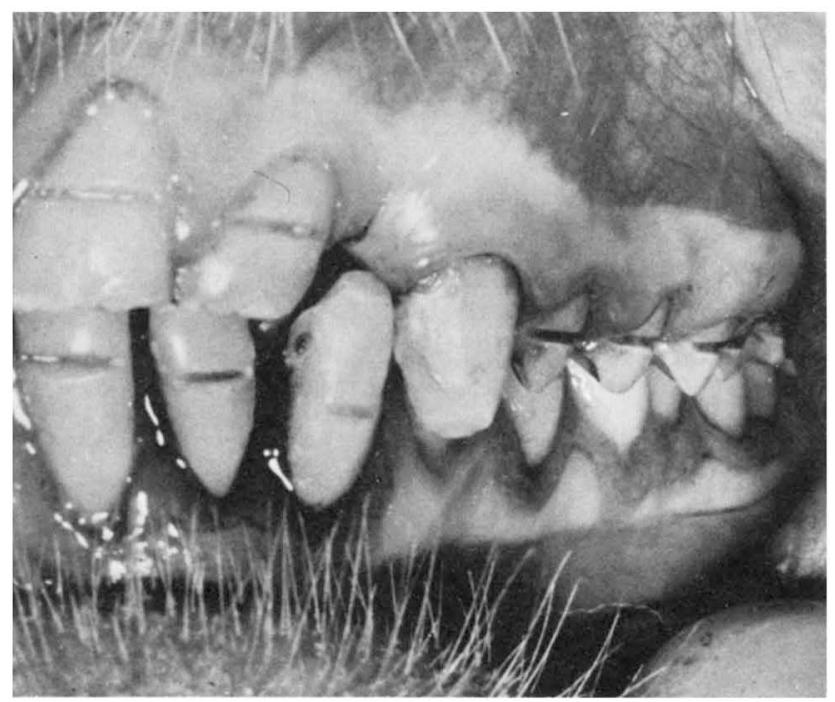

FIGURE 2. One month postoperatively. Note gingival inflammation and some recession in the quadrant of the surgery (right maxilla and left mandible). 
the control teeth, but an apical extension of the junctional epithelium was seen on several of the operated teeth.

Inflammation appeared to be more severe in the experimental than in the control quadrants.

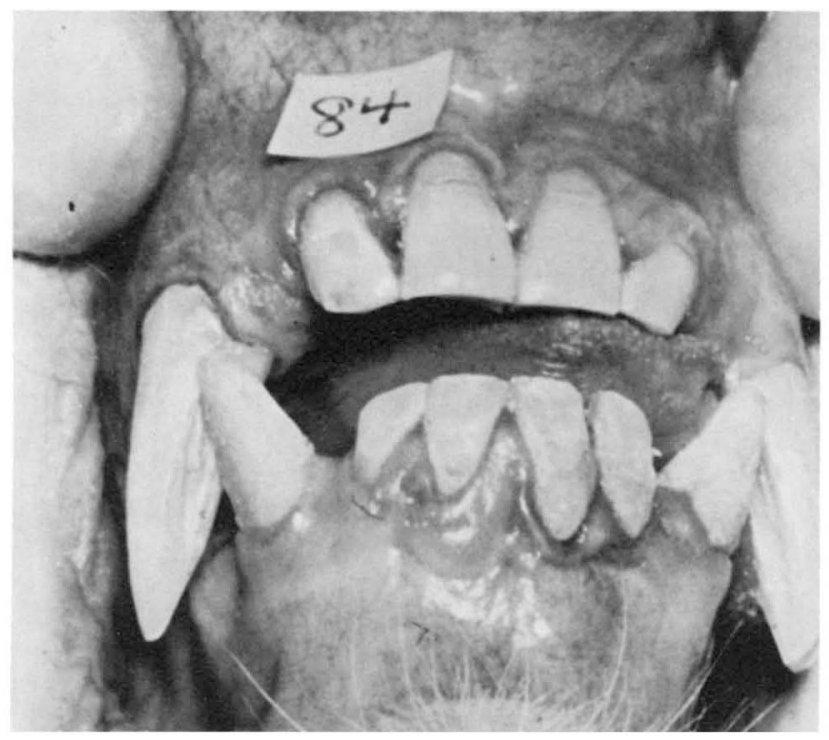

FIGURE 3. Six and a half months after surgery. Some gingival recession, more plaque, calculus and inflammation in the quadrants of the surgery (right maxilla and left mandible).
Local areas of root and bone resorption were observed in five experimental and three control teeth apparently related to trauma from occlusion rather than to the experimental procedures. Some areas of cemental resorption and repair were supracrestally located and cannot readily be explained except that they may be a result of accidental trauma against the metal cages housing the monkeys (Fig. 4A, and B).

Burn marks with cemental shrinkage and an increased staining potential were seen on some experimental root surfaces. The difference in staining occasionally extended through the cementum into the surface of the dentin.

Three Month Experimental Animal. Similar variations in attachment levels were observed for the 3 month animal as for the 1 month animal. In some instances there was complete healing with no lasting ill effect of the electrosurgery (Fig. 5). Junctional epithelium extended over and apically to burn marks on the roots of several experimental teeth.

On one tooth surface two distinct burn marks could be seen (Fig. 6). A few burn marks were observed in the dentin slightly coronally to the cemento-enamel junction indicating that the dentin had been affected through the enamel. Occasional burn marks were close to the alveolar crest but none was at the level of the crest.

Reactive secondary dentin was found in the pulp
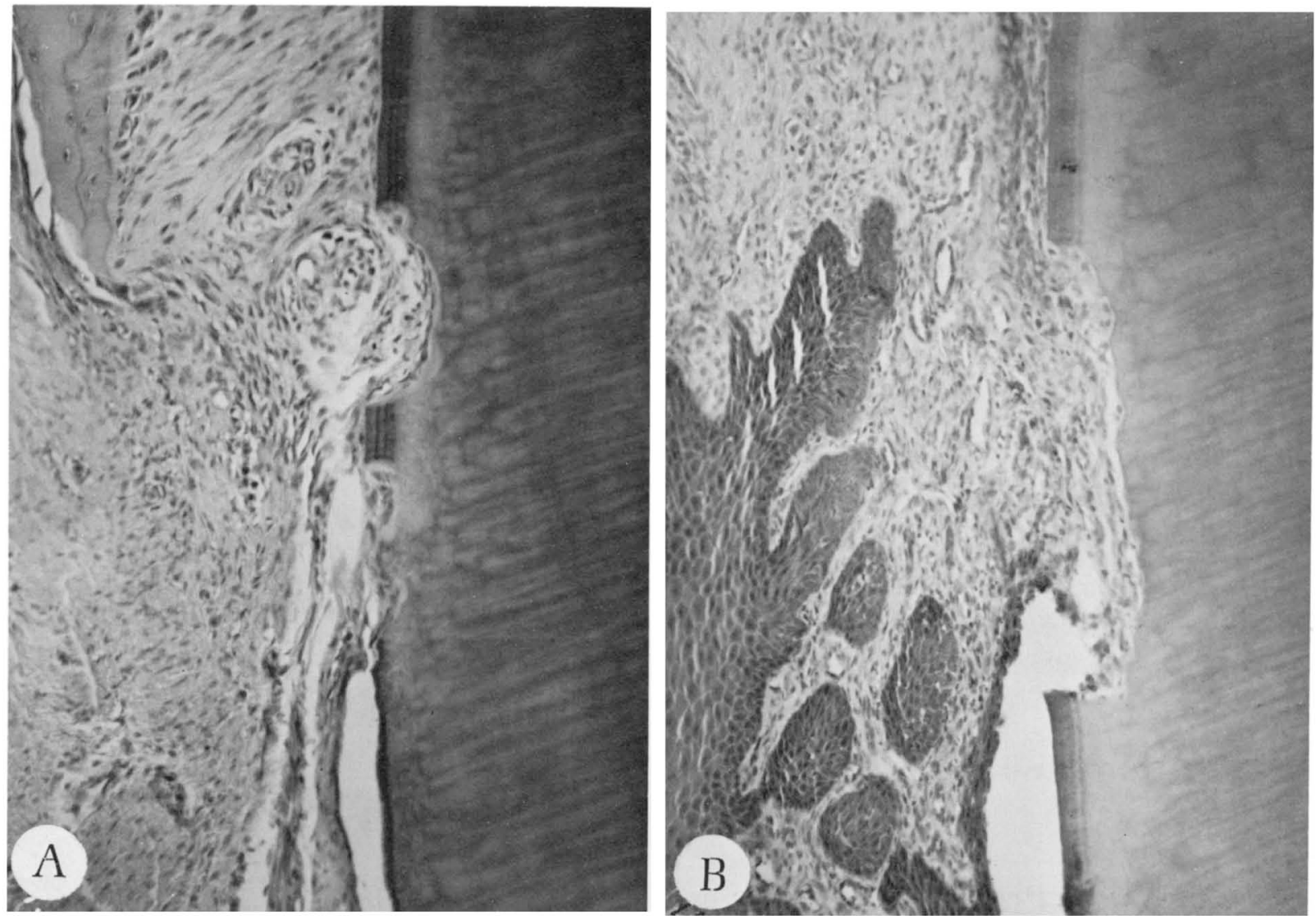

FIGURE 4A. One month experimental animal. Superficial root resorption involving supracrestal cementum and dentin on a tooth without surgery. (Original magnification $\times 195$ ). B. One month experimental animal. Superficial root resorption involving cementum and dentin apically to the junctional epithelium on a tooth with surgery. (Original magnification $\times 167$ ). 


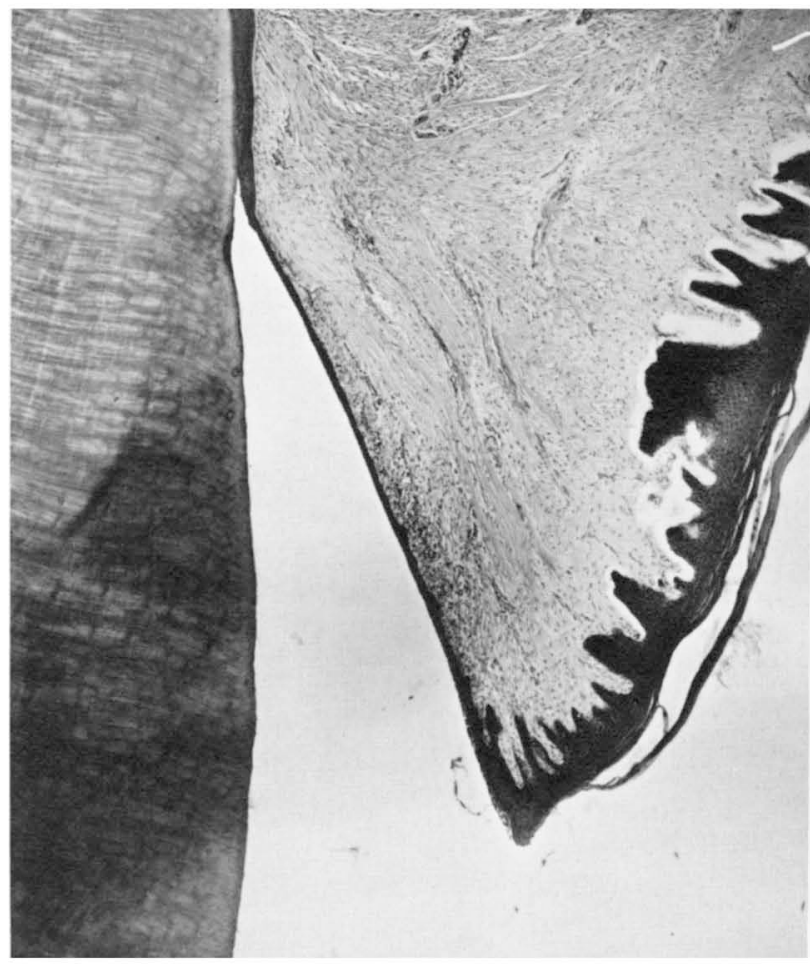

FIGURE 5. Three months experimental animal. Normal gingiva and the epithelial attachment ending at the cementoenamel junction on a tooth with surgery. (Original mangification $\times 46$ ).

chambers of five experimental teeth. It was distributed throughout the pulp chamber in some teeth whereas in others it was localized to areas corresponding to the prepared groove in the crown of the tooth, to a worn cusp tip or to burn marks near the cemento-enamel junction (Fig. 7).

Six and One-Half Month Experimental Animal. Long epithelial attachments on cementum were common. Gingival recession occurred both in control and experimental teeth.

Occasional burn marks covered by epithelium were observed apically to the junctional epithelium (Fig. 8). One tooth had two distinct burn marks and a long epithelial attachment (Fig. 9). Root resorption and shrinkage of cementum was found associated with burn marks and downgrowth of junctional epithelium (Fig. $10 \mathrm{~A}$ and $\mathrm{B}$ ). In a few instances a thin layer of cementum appeared over the burn marks (Fig. 10C).

Evidence of root resorption without relation to burns was seen on two experimental and two control teeth in the vicinity of the alveolar crest.

Secondary dentin appeared in five experimental and in one control tooth. In some teeth it was generalized throughout the pulp chamber and in one it was related to burn marks on the root.

Nine Month Experimental Animal. Several experimental teeth had normal attachment levels (Fig. 11) while others had long epithelial attachments. Several root surfaces showed burn marks usually covered by epithelium. A burn mark was also seen in dentin under

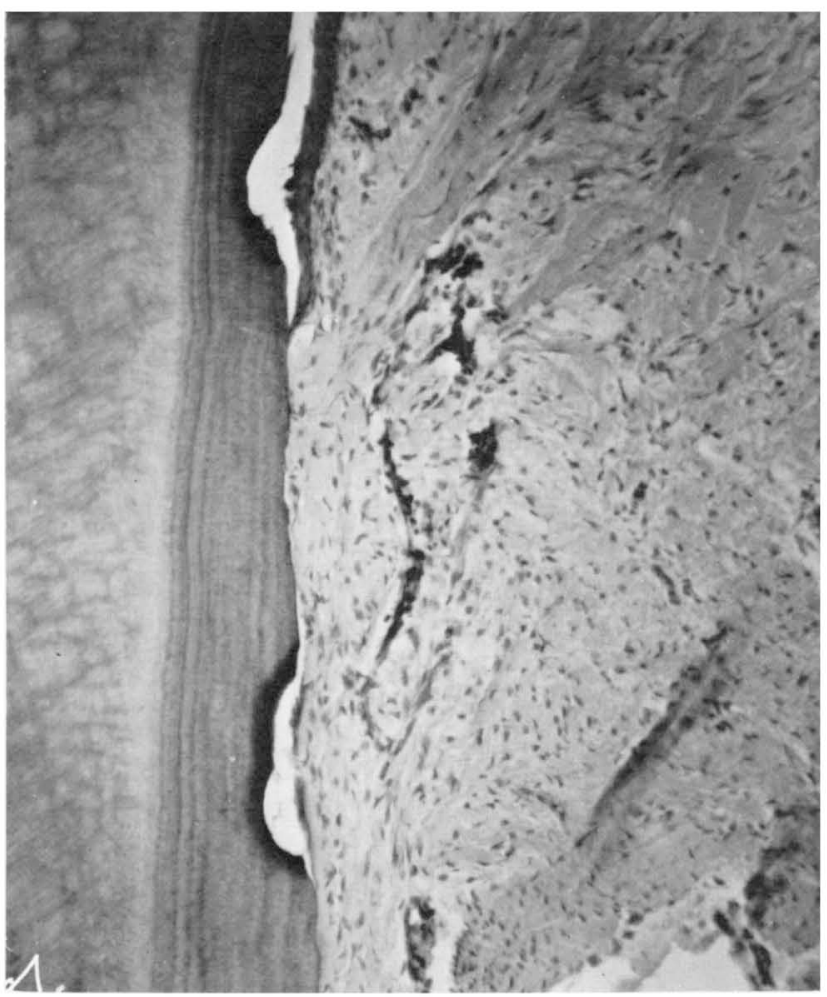

Figure 6. Three months experimental animal. Two burn marks from contact with the electrosurgical needle. The coronally placed mark was covered by junctional epithelium. Note the change in staining quality and the apparent loss of cemental substance associated with the burn. (Original magnification $\times 195$ ).

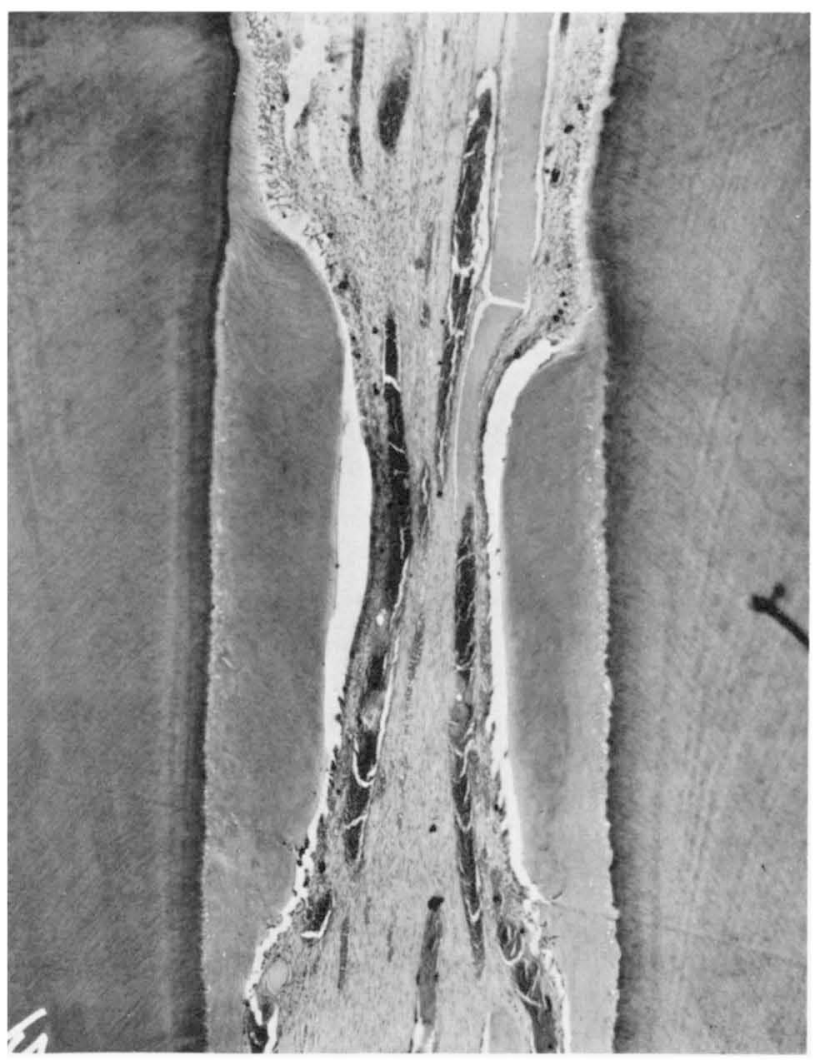

Figure 7. Three months experimental animal. Secondary dentin corresponding to electrosurgical contact with cementum on maxillary central incisor. (Original magnification $\times 42$ ). 
enamel close to the cementum-enamel junction (Fig. 12).

Generalized secondary dentin was seen in one experimental tooth. Two experimental teeth had root resorption in areas slightly coronally to the alveolar crest and without any evidence of burn marks.

\section{Biometric (Clinical) Data}

The means, standard deviation of the mean, and standard error, were determined for the pre and postoperative clinical measurements and all experimental and control teeth. The mean differences $(\bar{\Delta})$, standard deviation of the means (SD), and the standard error (SE), between the preoperative and postoperative measurements are presented in Table 1.

Significant differences $(P<0.001)$ between pre and postoperative clinical measurements (A and B) were found in the pairwise $T$ statistical analysis for experimental teeth. Analysis of similar pre and postoperative measurements on control teeth also showed significant $(P$ $<0.01$ ) differences.

Analysis of variance was done for measurements from the groove to the free gingival margin and from the groove to the bottom or base of the gingival crevice as well as for the following experimental variables: animals, experimental or control teeth, arch, and tooth position. The variable tooth position designates the position of a boin in relation to other teeth in that or other quadrants. The variabies include second molar, first molar, second risuspid, latera! ins:ser and central incisor. The teeth

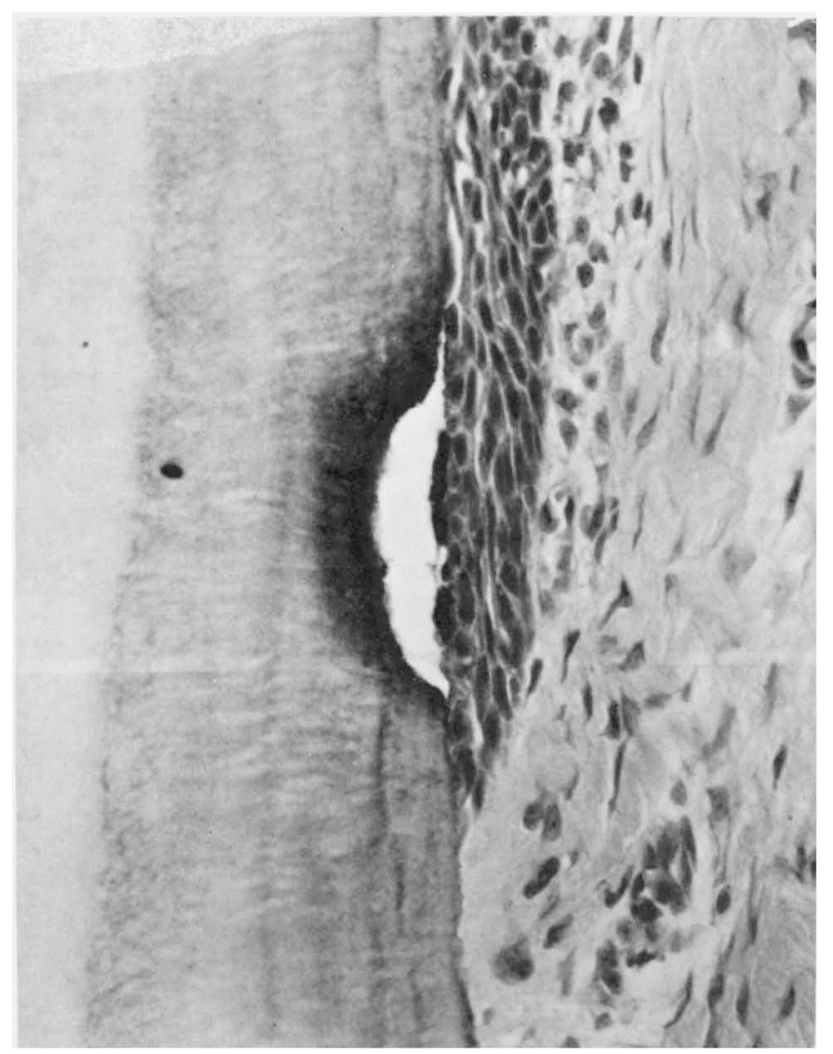

FIGURE 8. Six and a half months experimental animal. Epithelial extension over burn mark from contact with the electrosurgical needle. Mandibular incisor. (Original magnification $\times$ 465).

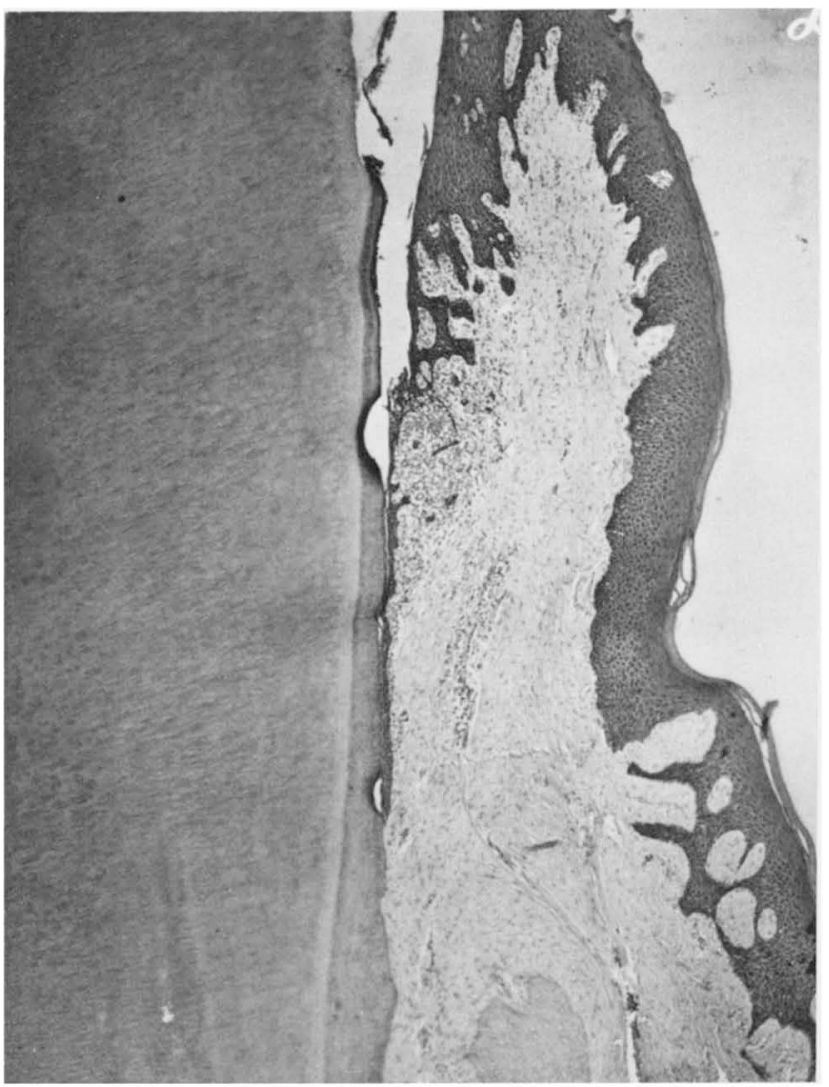

Figure 9. Six and a half months experimental animal. Long epithelial attachment extending over two burn marks on the cementum on mandibular incisor. The coronally situated burn mark extends through the entire cemental covering. (Original magnification $\times 46$ ).

were grouped according to anatomical location in a quadrant without respect to arch or the side of the mouth on which they are located. The sum of squares, mean squares, $F$ statistic, degree of freedom and significance were computed for selected and related variables.

The analysis of variance for the measurement from the groove to the free gingival margin demonstrated a significant difference between experimental and control teeth $(P<0.001)$. A significant difference was also found when the experimental variables tooth position and experimental or control teeth were analyzed $(P<0.01)$. The greatest differences between pre and postoperative measurements occurred on the central and lateral incisors whereas the smallest differences were seen on the molars.

No significant experimental animal difference was found when the experimental variables animal and experimental or control teeth were analyzed. Also no significant differences were seen between arches when this experimental variable and experimental or control teeth were analyzed.

While a significant difference was found for tooth position, this did not seem to be related to the arch in which the tooth was located. This was suggested by the finding of no significant difference for analysis of the variables tooth position, arch and experimental or control teeth. 

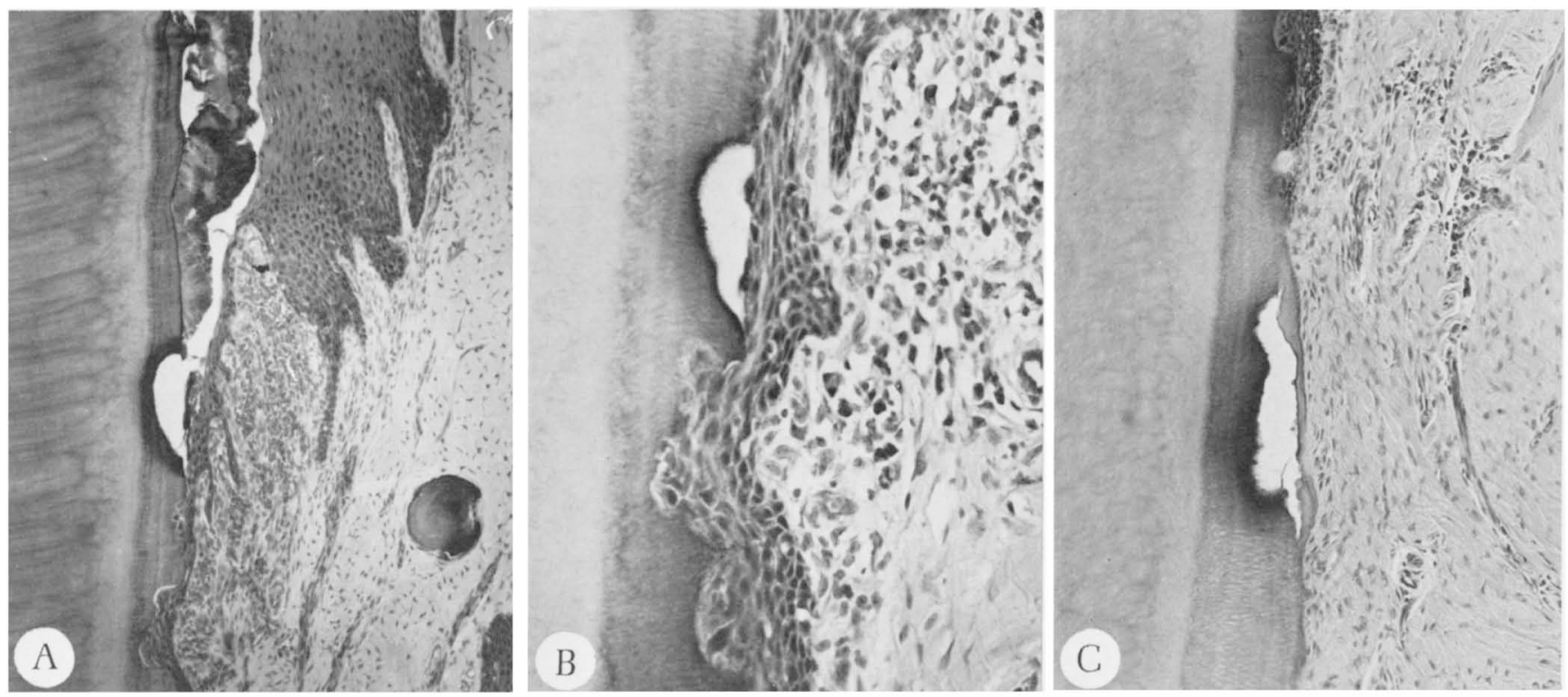

FIGURE 10A. Six and a half months experimental animal. Note deep burn mark through cementum at the middle of the picture. Superficial resorption of cementum covered by epithelium further apically (bottom of picture). (Original magnification $\times 132$ ). B. Higher magnification from middle part of Figure $10 \mathrm{~A}$ showing epithelial adaptation to area of cemental resorption, while a void appears between the epithelium and the cementum at the burn mark. This void may be an artifact due to removal of mineral salts during decalcification in an area devoid of organic matrix as a result of surgery. (Original magnification $\times 406$ ). C. Another section from the same tooth as Figures $10 \mathrm{~A}$ and $10 \mathrm{~B}$. Note new cementum formed over burn mark and void at middle of picture. This also supports the assumption that the void is an artifact following loss of calcified material lacking organic matrix during decalcification of the specimen. (Original magnification X155).

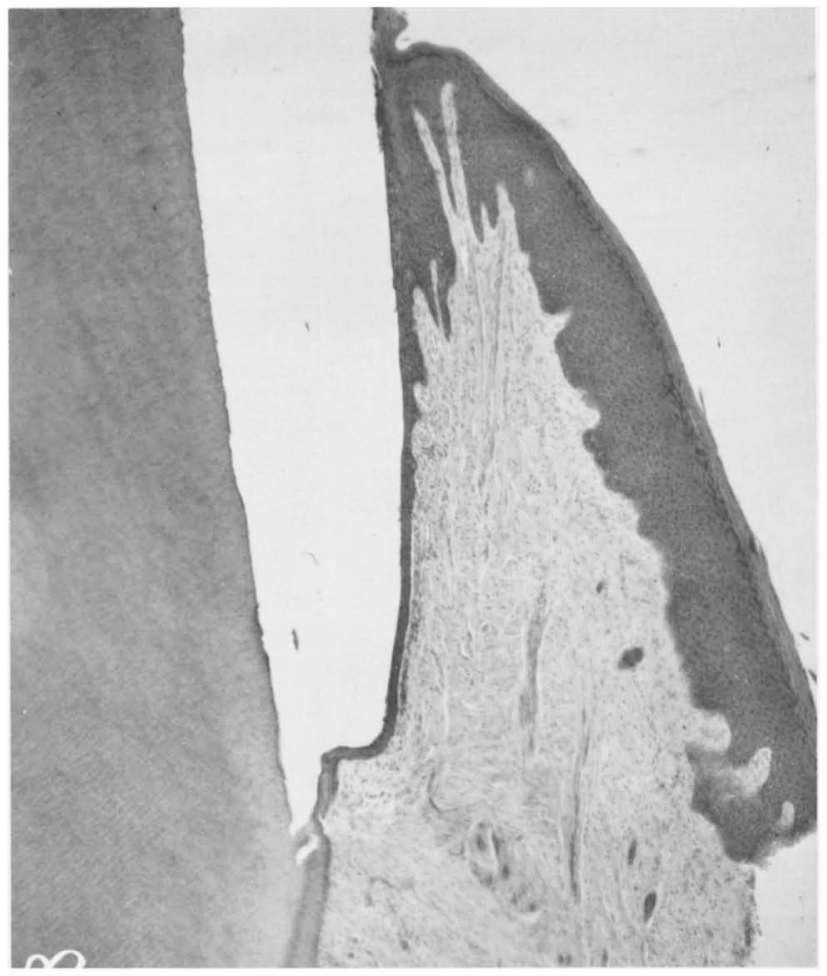

FIGURE 11. Nine months experimental animal. Normal gingiva with the epithelial attachment at the cemento-enamel junction on a tooth exposed to electrosurgery.

The analysis of variance for measurements from the groove to the bottom of the gingival crevice, also demonstrated a significant difference between experimental and control teeth $(P<0.001)$. No significant difference was found related to tooth position when the experimental variables tooth position and experimental or control teeth were analyzed.

A significant experimental animal difference was found when the variables animal and experimental or control teeth were analyzed $(P<0.05)$. Because sample size was small and there appeared to be no relationship between mean values and the duration of the healing period, no conclusions were drawn regarding animal differences and time allowed for healing.

As was also seen with the measurements to the free gingival margin no significant differences were found between arches when this experimental variable and experimental and control teeth were analyzed. The analysis of measurements from the groove to the bottom of the gingival crevice also demonstrated no significant difference for the experimental variables tooth position, arch and experimental or control teeth.

\section{Histometric Data}

The sample numbers, means, standard deviations of the means (SD) and standard error (SE) were determined for histometric measurements for experimental and control teeth (Table 2).

Incomplete histometric data were available for some teeth mainly because the grooves had not been cut deep enough into the dentin. The sample numbers for some statistical analyses were reduced because only teeth with the required data could be included.

Analysis of variance was done for the linear measurements indicated in Figure 1. The statistical analysis also included the experimental variables; animal, experimen- 
tal or control teeth, arch and tooth position (second molar, first molar, second bicuspid, lateral incisor or central incisor).

The 9 month animal could not be included in the analysis of variance for histometric data because several sections were lost in preparation. The omission was made because empty cells were undesirable in analysis of variance. Thus this analysis was based upon data derived from the 1,3 , and $6 \frac{1 / 2}{2}$ month animals.

Significant experimental animal difference was found for only one measurement $[(F)$ cemento-enamel junction to the connective tissue border of the most apically located epithelial cells on the lingual root surface]. Because sample size was small and there appeared to be no relationship between mean values and the duration of

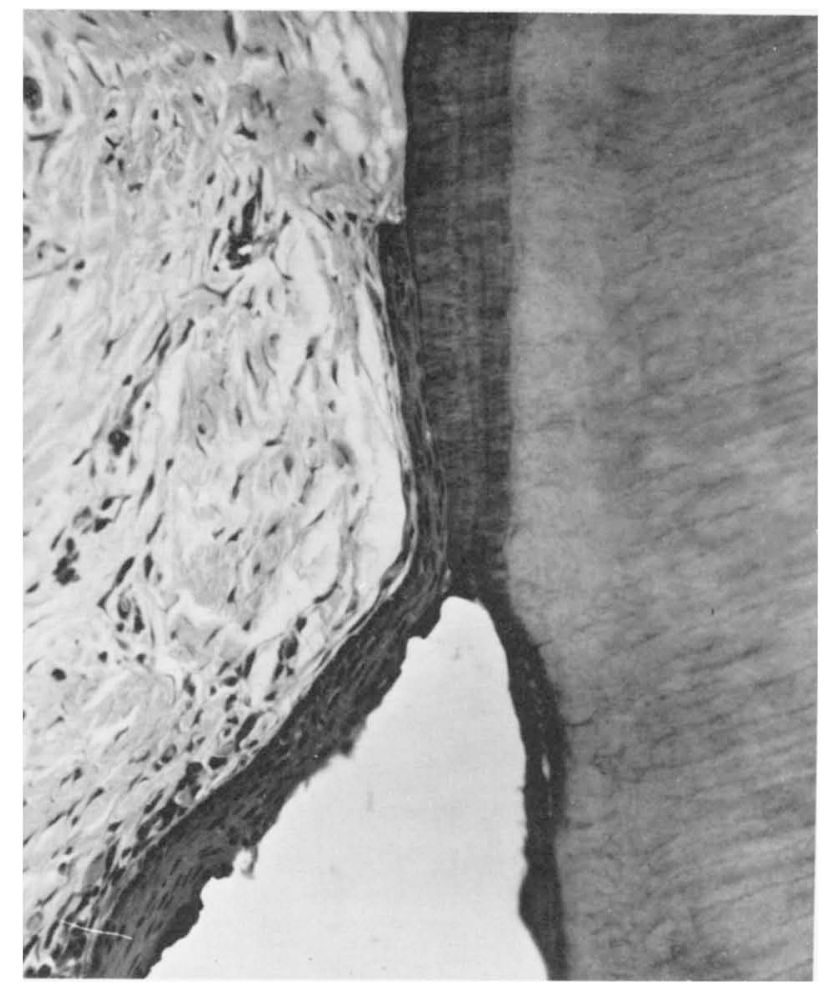

FigurE 12. Nine months experimental animal. Note superficial burn mark in dentin slightly coronal to the cemento-enamel junction indicating penetration of the effect of the electrosurgery through the thin enamel in this area. Otherwise normal junctional epithelium extending for a short distance over cementum. (Original magnification $\times 340$ ). the healing period, no conclusions were drawn regarding animal differences and time allowed for healing.

There were significant differences $(P<0.01)$ with variables experimental and control teeth for measurements $(D, F$, and $D+F$, from Figure 1) which relate cemento-enamel junction to the most apically located epithelial cells on the root surface. No significant differences were found for $E$ and $G$ which measure the distance from the cemento-enamel junction to the alveolar crest. When the linear measurements $(E+G)$ were summed, a significant difference was found $(P<0.05)$. It appears that the location of epithelial cells on the root surface was influenced more by the electrical gingival resection than was the level of the alveolar crest in these analyses in which the experimental time period was limited to $6 \frac{1 / 2}{2}$ months.

In the analysis of variance no significant differences were seen for arch or tooth position with regard to the histometric measurements.

Pairwise $\mathrm{T}$ statistics including mean, standard deviation of the mean, $T$ statistic and significance for selected pairs of linear measurements demonstrated a significant difference $(P<0.05)$ between the facial and lingual surfaces for the cemento-enamel junction to the most apically located epithelial cell measurements for the experimental teeth. There was no significant difference for the control teeth.

The cemento-enamel junction to alveolar crest measurement for experimental teeth also showed a significant difference $(P<0.05)$ between facial and lingual surfaces. Similar measurements were not significantly different for the control teeth.

Greater change in the levels of the crevicular epithelium and alveolar crest occurred on the facial than the lingual surfaces.

\section{Comparison of Biometric and Histometric Data}

The postoperative clinical measurements of groove to bottom of gingival crevice were compared with the histometric measurements of groove to the connective tissue border as located by the most apically positioned epithelial cells on the root surface.

The pairwise $\mathrm{T}$ statistical analysis showed a significant difference $(P<0.001)$ between the clinical and histometric values for the experimental teeth. For the control

TABLE 1. Sample Number, Mean in Millimeters, Standard Deviation of the Mean and Standard Error for the Mean Difference $(\bar{\Delta})$ between Preoperative and Postoperative Measurements of Experimental and Control Teeth

\begin{tabular}{lllll}
\hline Measurement & N & Mean $(\bar{\Delta})$ SD & SE \\
\hline Groove to FGM (A) & & & & \\
\hline Experimental teeth & 44 & .96 & .560 & .084 \\
$\quad$ Control teeth & 44 & .14 & .332 & .050 \\
Groove to Base (B) & & & & \\
Experimental teeth & 44 & 1.15 & .678 & .102 \\
Control teeth & 44 & .16 & .369 & .056 \\
\hline
\end{tabular}


TABLE 2. Sample Number, Mean in Millimeters, Standard Deviation of the Mean and

Standard Error for Histometric Measurements on Combined Facial and Lingual Surfaces of Experimental and Control Teeth

\begin{tabular}{ccccc}
\hline Measurement & $\mathrm{N}$ & Mean & SD & SE \\
\hline CEJ to Base (D\&F) & & & & \\
\hline $\begin{array}{l}\text { Experimental teeth } \\
\text { Control teeth }\end{array}$ & 42 & .83 & .747 & .115 \\
CEJ to Crest (E\&G) & 44 & .32 & .334 & .050 \\
$\quad \begin{array}{ll}\text { Experimental teeth } \\
\text { Control teetn }\end{array}$ & 42 & 1.55 & .805 & .124 \\
\end{tabular}

TABle 3. Correlation Coefficients ( $r$ ) between Clinical Measurement (B) Groove to Bottom of Gingival Crevice and Histometric Measurement (C) Groove to the Most Apically Located Epithelial Cells

\begin{tabular}{ccccc}
\hline Variable & N & r & p & $\begin{array}{c}998 \text { Confidence } \\
\text { Limits }\end{array}$ \\
\hline Experimental teeth & 19 & .8994 & .01 & $.69--.98$ \\
Control teeth & 25 & .8636 & .01 & $.65--.96$ \\
\hline
\end{tabular}

teeth, there was no significant difference. For the experimental and control teeth the clinical measurements were larger than the histometric measurements. Significant correlation (Table 3$)$ was found at $(P<0.01)$ between similar postoperative clinical and histometric measurements both for experimental and control teeth.

\section{SigNifiCANCE}

Previous studies have established that electrosurgery done by fully rectified current, properly adjusted, used with the appropriate rate of motion, and a fine single filament electrode produces a very narrow zone of soft tissue destruction which heals in a way similar to a surgical incision with a knife. ${ }^{5,6,12}$ However, if contact with calcified structures is included in the incision, altered healing patterns have been reported. Of special interest is the report by Ozimek, ${ }^{17}$ who found that electrosurgical incisions contacting periosteum or tooth structure resulted in delayed healing. Structural alterations were observed both in the lamina propria and in the contacted calcified tissues. He suggested that increased resistance of a fully rectified high frequency current may have resulted in degradation yielding a blended coagulation and cutting current which produced coagulation rather than volatilization of cells.

The literature is replete with advice regarding proper type of current, proper current density, appropriate size and shape of electrode, consistent rate of electrode movements, clean electrodes, dry field and healthy tissues prior to the electrosurgical procedure. Every article warns against the violation of the gingival attachment and most articles stress the importance of staying within the gingival crevice. ${ }^{18}$ However, it is very difficult to perform electrosurgery in the gingival crevice without accidental or incidental contact with the tooth surface, and to assume that probing of a gingival crevice extends only to the coronal aspect of the epithelial attachment is a serious misconception.

It has been shown, both by previous investigations ${ }^{19,} 20$ and the present study that routine crevicular probing extends through the epithelial attachment and ends at the coronal aspect of the connective tissue attachment. Probing actually has a tendency to extend slightly into the connective tissue attachment which explains that when the incision in the present study was made to the level located with probing, there was a tendency for the incision to extend beyond the epithelial attachment and into the connective tissue attachment.

A serious hazard of electrosurgery is the apparent alteration of the surface of the cementum in areas contacted with an activated electrosurgical needle. In some instances this alteration even extended into the dentin and where the enamel was thin close to the cemento-enamel junction, the burn extended through the enamel and into the dentin. The cemental surfaces that had been altered by the current were not conducive to reparative cementogenesis. The burn areas usually were covered by epithelium or occasionally had initiated resorptive processes. In a few instances where the alteration had been minimal, the burn marks were covered by new cementum (Fig. 10C).

It appeared in general that where the needle had contacted only enamel, there was no permanent injury to the connective tissue attachment to the teeth and new epithelial aitachment to enamel took place. However, in areas where the epithelial attachment extends over 
cementum, which it very commonly does both in adult monkeys and humans, there is a great potential for the electrosurgical needle to touch cementum when the incision is aimed at the bottom of the crevice as located by probing.

One area of sequestration of the alveolar process was observed. Obviously in this instance the electrosurgical needle inadvertently had come too close to the alveolar process. It has to be realized that the distance between the crevice and the bone is shorter in monkeys than in humans so the chance for such an error to occur is greater in monkeys than it would be in human patients. However, it should serve as a warning regarding the potential danger of operating the electrosurgical unit close to the alveolar process.

In the absence of oral hygiene more plaque and calculus accumulated on the operated than on the control teeth, probably because of ulceration interfering with normal self-cleansing function. The postsurgical inflammation related to increased plaque formation may have been responsible for some delay in gingival healing. However, the loss of attachment was so clearly related to the electrosurgical injury to the cementum that the loss of attachment cannot be explained on the basis of the increased plaque accumulation.

A slight loss of alveolar crestal bone occurred for the experimental teeth; however, this loss was not statistically significant for the facial or the lingual surfaces taken separately. When these data were pooled a statistically significant difference $(P<0.05)$ was found. The data suggests that the alveolar crest was altered less by electrical gingival resection than the position of the free gingival margin and the connective tissue attachment to the teeth. The greatest recession of the gingival margin occurred around the anterior teeth where the gingiva is thin. The difference in gingival recession related to tooth position was statistically significant $(P<0.01)$.

The loss of attachment with electrosurgery was not significantly related to tooth position.

Comparison of the biometric and histometric measurements of the distances from the cemento-enamel junction to the coronal aspect of the connective tissue attachment, verified previous observations ${ }^{19,}{ }^{20}$ that clinical probing tends to extend to the connective tissue attachment rather than to end at the coronal aspect of the epithelial attachment. There was a statistically significant correlation $(P<0.01)$ between clinical and histometric measurements (B and C Table 3 ) from the groove to the bottom of the sulcus and from the groove to the most apically located epithelial cells on the root surface for both experimental $(r=.89)$ and control teeth $(r=.86)$.

The findings in this study indicate that "troughing" of the gingival crevice with electrosurgery should be done with great care and definitely should not be extended to the bottom of the crevice as located by probing. The study verified the finding of Glickman and Imber ${ }^{14}$ that no permanent damage occurs from electrosurgery so long as the surgery is confined to the free gingiva and the needle only contacts enamel, not too close to the cemento-enamel junction. It should be emphasized that in persons of age 25 to 30 years or older, the epithelial attachment almost always extends over cementum with a potential for harmful contact between the electrode and the cementum. The nature of the cemental lesion produced by electrosurgery should be investigated further. The reason for the open spaces between the electrosurgical burn and the covering epithelium seen in the photomicrographs is not readily explainable, but is probably an artifact due to loss of mineral salts during decalcification in an area devoid of collagen matrix following the electrosurgical burn. The abundant secondary dentin formation related to electrosurgical contacts with the root surfaces was an interesting side finding which may explain observations of the desensitization of teeth with exposed root surfaces by moving an activated electrode over the surfaces and it confirmed previously reported findings. ${ }^{21}$

\section{SUMMARY}

A biometric and histometric study of electrosurgical gingival "troughing" with a fully rectified current was accomplished in four Rhesus monkeys. The operation resulted in a statistically significant recession of the free gingival margin and loss of connective tissue attachment associated with apical migration of the junctional epithelium. Burn marks from contacts with the electrodes were observed on the cemental surfaces and in the dentin under the enamel close to the cemento-enamel junction. The cemental burn marks usually were covered by epithelium, which may explain the apical migration of the junctional epithelium observed in the experimental teeth. A slight loss of crestal alveolar bone occurred in the experimental areas and a bone sequestrum formed in one instance. Secondary dentin was found in response to electrosurgical contact of the cemental surface.

\section{CONCLUSIONS}

1. Electrosurgical gingival troughing in Rhesus monkeys resulted in: (a) significant recession of the free gingival margin, (b) apical positioning of the sulcular epithelium, (c) loss of some connective tissue attachment, (d) burn marks on root surfaces where contact was made with the electrode, and (e) slight loss of crestal alveolar bone.

2. Electrosurgery should be confined to the free gingiva without making contact with cementum or bone.

3. Gingival electrosurgery for crevicular troughing involves a considerable risk of producing permanent periodontal damage.

\section{ACKNOWLEDGMENT}

The histological sections were prepared by Jean I. Simons at the Veterans Administration Hospital, Ann Arbor, Michigan.

REFERENCES

1. Malone, W., and Manning, J.: Electrosurgery in restorative dentistry. $J$ Prosthet Dent 20: 417, 1968 
2. Oringer, M.: Electrosurgery for definitive conservative modern periodontal therapy. Dent Clin North Am 13: 53, 1969.

3. Nuckles, D.: Electrosurgery as an aid to crown and bridge procedures. J SC Dent 30: 7, 1972.

4. Kelly, W.: Gingival Tissue Regeneration Following Electrosection. 62 p typed thesis. St. Louis University, 1968.

5. Malone, W., Eisenmann, D., and Kusek, J.: Interceptive periodontics with electrosurgery. J Prosth Dent 22: 555, 1969.

6. Eisenmann, D., Malone, W., and Kusek, J.: Electronmicroscopic evaluation of electrosurgery. Oral Surg 29: 660, 1970.

7. Aremband, D., and Wade, A.: A comparative wound healing study following gingivectomy by electrosurgery and knives. J Periodont Res 8: 42, 1973.

8. Armstrong, S., Podshadley, A., Lundeen, H., and Scrivner, E.: The clinical response of gingival tissues to electrosurgical displacement procedures. J Tenn $S$ Dent $A$ 48: $271,1968$.

9. Schieda, J., DeMarco, T., and Johnson, L.: Alveolar bone response to the electrosurgical scalpel. J Periodontol 43: 225,1972

10. Klug, R.: Gingival tissue regeneration following electrical retraction. J Prosthet Dent 16: 955, 1966.

11. Schneider, A., and Zaki, A.: Gingival wound healing following experimental electrosurgery. A light microscopic and macroscopic investigation. J Periodontol 45: 459, 1974.
12. Schneider, A., and Zaki, A.: Gingival wound healing following experimental electrosurgery: An electronmicroscopic investigation. J Periodontal 45: 685, 1974.

13. Pope, J., Gargiulo, A., Steffileno, H., and Levy, S.: Effects of electrosurgery on wound healing in dogs. Periodontics 6: 30, 1968.

14. Glickman, I., and Imber, T.: Comparison of gingival resection with electrosurgery and periodontal knives. A biometric and histologic study. J Periodontol 41: 142, 1970.

15. O'Leary, T., Standish, S., and Bloomer, R.: Severe periodontal destruction following impression procedures. $J$ Periodontol 44: 43, 1973.

16. Ramfjord, S. P.: Gingivectomy-Its place in periodontal therapy. J Periodontol 23: 30, 1952.

17. Ozimek, J.: Comparative Healing of Electrosurgical and Scalpel Inflicted Incisional Wounds. $60 \mathrm{p}$ typed thesis. Maywood, Loyola University, 1972.

18. Malone, W. F.: Electrosurgery in Dentistry. Springfield, Ill., Charles C. Thomas Publishers, 1974.

19. Kohler, C., and Ramfjord, S.: Healing of gingival mucoperiosteal flaps. Oral Surg 13: 89, 1960.

20. Sivertson, J. F.: Probing of Pockets Related to the Attachment Level. v $+35 \mathrm{p}$ typed thesis. Ann Arbor, The University of Michigan, 1974

21. Agnew, R., and Kaiser, W.: Effects upon the dental pulp of the macacus rhesus of externally applied high frequency electrosurgical currents. Abstract no. 499, I.A.D.R., 1952.

\section{Abstracts}

The Feasibility of A Screening Procedure Regarding TEMPoromandibular JoINT DySFUnCTION (TMJ)

Rosenbaum, M.

Oral Surg 39: 382, March, 1975.

The question as to whether muscle tenderness to palpation had any relation to TMJ dysfunction was examined in 279 patients using a bimanual palpation technique for the internal pterygoid, external pterygoid and the masseter muscles. Pain was reported upon palpation in 75 patients many of which were either totally or partially edentulous and wore dentures. There was no statistical correlation between tenderness upon palpation and other clinical signs of TMJ dysfunction such as headache, dizziness, crepitus, or maximum opening. 2 Howes Avenue, Stamford, Connecticut 06906

\section{Bovine Gingival Collagenase: Demonstration and Initial Characterization}

Birkedal-Hansen, H., Cobb, C. M., Taylor, R. E., and Fullmer, H. M. J Oral Pathol 3: 232, No. 5, 1974.

Gingiva was obtained from young cattle, treated with antibiotic solutions, thinly sliced, and cultured on blood agar plates. Culture fluids were harvested either daily or every 3rd day. Collagenase activity of the precipitate was assayed and shown to be continually produced for at least the 30 days studied. This collagenase was inhibited by human serum, by ethylenediamine tetraacetic acid, and by cysteine, showing properties similar to human collagenase. During the 30-day period the connective tissue gradually disappeared. Institute of Dental Research, Birmingham, Alabama 35294
In Vivo Calcification of Implanted Bone Cells

McKinney, R. V., Dirksen, T. R., Schuster, G. S., and Bustos, S. J Oral Pathol 3: 256, No. 5, 1974.

Twenty-four hours after birth new born rats were decapitated, and bone cells isolated from the frontal, parietal and occipital bones were prepared for culture. Chambers containing suspensions of calvaria cells were implanted subcutaneously into four rats of the same species. The animals were sacrificed at intervals of $42,68,118$, and 279 days. The results showed that in the 118-day specimens a substance that was suggestive of osteoid material was present. The 279-days specimen revealed material that was compatible to calcified membranous tissue. Department of Oral Pathology, School of Dentistry, Medical College of Georgia, Augusta, Georgia 30902

Clinical Evaluation of Free Connective Tissue Grafts Used TO INCREASE THE WidTH OF KeRATINIZED Gingiva

Edel, A.

J Clin Periodont 1: 185, No. 4, 1974.

Utilizing gingival connective tissue excised by three different procedures, 14 sites in eight subjects were grafted to determine the applicability of connective tissue grafts to mucogingival procedures. Preparation for surgery included scaling and oral hygiene instruction. The width of the gingiva from gingival margin to mucogingival junction was measured preoperatively and at intervals during the 6-month study period. A statistically significant increase in the width of attached gingiva was demonstrated. 59 Fryent Way, Kingsbury, London N.W. 9 $9 N U$, England 